\title{
Stellar model atmospheres with abundance stratification
}

\author{
F. LeBlanc ${ }^{1,2}$, D. Monin ${ }^{3}$, A. Hui-Bon-Hoa ${ }^{2}$, and P. H. Hauschildt ${ }^{4}$ \\ 1 Département de Physique et d'Astronomie, Université de Moncton, Moncton, NB E1A 3E9, Canada \\ e-mail: francis.leblanc@umoncton.ca \\ ${ }^{2}$ Laboratoire d'Astrophysique de Toulouse-Tarbes, Université de Toulouse, CNRS, 14 avenue Édouard Belin, 31400 Toulouse, \\ France \\ 3 Dominion Astrophysical Observatory, Herzberg Institute of Astrophysics, National Research Council of Canada, 5071 West Saanich \\ Road, Victoria, BC V9E 2E7, Canada \\ ${ }^{4}$ Hamburger Sternwarte, Gojenbergsweg 112, 21029 Hamburg, Germany
}

Received 22 August 2008 / Accepted 19 December 2008

\section{ABSTRACT}

\begin{abstract}
Context. Atomic diffusion is believed to be an important physical process in the atmospheres of several types of stars. Stellar atmospheres, including the stratification of the elements due to diffusion, are then needed to properly compare theoretical results to observations for such stars.

Aims. This paper aims to estimate the effect of vertical abundance stratification on the atmospheric structure of stars and its potential importance regarding observational anomalies for various types of stars.

Methods. Simulations using a modified version of the PHOENIX atmosphere code will be described, while taking vertical abundance stratification into account.

Results. Our results show that large abundance gradients can exist in the atmospheres of Ap and blue horizontal branch stars. Stratification can also lead to relatively large atmospheric structural changes. The effect of elemental stratification on the atmospheric structure might well be able to explain the well-known core-wing anomaly of the Balmer lines observed for cool Ap stars.
\end{abstract}

Key words. stars: atmospheres - stars: abundances - stars: chemically peculiar

\section{Introduction}

Microscopic diffusion in hydrodynamically stable stellar regions can lead to abundance stratification (Michaud 1970). If diffusion is the dominant transport process in the stellar atmosphere, vertical abundance gradients can occur and can modify its structure (Hui-Bon-Hoa et al. 2000; LeBlanc \& Monin 2004). The diffusion phenomenon is due to the competition of the various forces on the atomic species (e.g. radiative, gravitational, electrical, etc.). The main ingredient in the diffusion equation is the radiative acceleration term, which is related to the momentum transferred to ions from the radiative field during photoexcitation or photoionization (e.g. Gonzalez et al. 1995). Accurate computation of radiative accelerations necessitates precise knowledge of atomic data, as well as the local physical conditions, especially the radiative flux.

In the past, the vast majority of spectroscopic and photometric studies have employed stellar atmospheres with vertically uniform abundances. Recent theoretical studies of white dwarfs (Dreizler \& Wolff 1999) and blue horizontal branch (hereafter BHB) stars (Hui-Bon-Hoa et al. 2000) have attempted to remedy this situation by calculating self-consistent stellar atmospheres including vertical abundance gradients caused by diffusion. Self-consistent models are defined here as atmospheres whose structure is calculated with the stratification of the elements predicted by diffusion. These models have been able to explain certain observational anomalies caused by radiative diffusion in the atmosphere of these types of stars. Recently, Alecian $\&$ Stift (2007) has calculated abundance stratification in the atmospheres of magnetic Ap stars. However, these last models do not take the effect of stratification on the atmospheric structure into account. Meanwhile, Shulyak et al. (2004) constructed model atmospheres that empirically include stratification of the elements. These are LTE models that treat the opacities using the line-by-line method.

Meanwhile, Michaud et al. (2008) have constructed evolutionary models for BHB stars. These models do not treat the atmosphere in detail and assume that it is chemically homogeneous. However, recent observational evidence (Khalack et al. 2007 and 2008) shows that the atmospheres of such stars exhibit vertical abundance stratification (see discussion below).

Observational evidence of the existence of vertical abundance gradients is mounting for several types of chemically peculiar (CP) stars (e.g. Ryabchikova et al. 2003; Ryabchikova 2005). Abundance stratifications for several elements have been detected in Ap stars. For example Babel (1994) detected Ca stratification in such stars, while Wade et al. (2001) showed that strong and weak lines of certain iron peak elements could not be fitted by a single abundance. They also showed that an empirical two-zone abundance profile could reproduce spectra better. Many other studies pertaining to the detection of stratification in the atmospheres of Ap stars have also been undertaken (e.g. Bagnulo et al. 2001; Kochukhov et al. 2006; Ryabchikova et al. 2002, 2005 and 2008). The method commonly used to detect abundance stratification in Ap stars is based on finding an abundance stratification profile that fits better the atomic lines.

Stratification of $\mathrm{N}$ and $\mathrm{S}$ has also been detected in the BHB star HD135485 (Khalack et al. 2007). Stratification of other metals, including iron, has also been detected in several other BHB stars (Khalack et al. 2008). Since abundance 
stratification of Fe is found in several BHB stars, the evidence of the presence of an abundance stratification in the atmospheres of this type of stars is quite convincing. The approach used for the detection of elemental stratification in BHB stars differs from the one used in the references given above related to Ap stars. Abundance stratification signatures are detected by calculating the abundance of lines formed at different depths (while assuming an homogeneous abundance during these calculations).

Stratification of $\mathrm{Cr}$ in $\mathrm{HgMn}$ stars has also been suggested in an observational analysis by Savanov \& Hubrig (2003). Recently, Thiam et al. (2008) detected signatures of Mn stratification in the HgMn star HD178065. Moreover, Sigut et al. (2000) suggest that the occurrence of emission lines in the ${ }^{3} \mathrm{He}$ star 3 Cen $\mathrm{A}$ is a sign that abundance stratification occurs in the atmosphere of this type of CP star. However, more observational studies of $\mathrm{HgMn}$ stars are needed to confirm the presence of elemental stratification in their atmosphere.

For a better understanding of these CP stars, it is imperative to have stellar atmosphere models that include self-consistently such abundance gradients.

In this paper we will show results of self-consistent atmospheric modeling including vertical abundance stratification for two stellar models with $T_{\text {eff }}=8000$ and $12000 \mathrm{~K}$, respectively. These models were chosen since they are typical of Ap and BHB stars, in which diffusion is most likely important in their atmosphere. Atmospheres of BHB stars with $T_{\text {eff }} \geq 11500 \mathrm{~K}$ have low rotational velocities and thereby are hydrodynamically stable. Meanwhile, Ap stars possess large magnetic fields and it is commonly believed that these completely inhibit convection. Below, we will show the effect of one aspect of the magnetic field on the diffusion of the elements. Our models seek to obtain a vertical abundance profile that is at equilibrium (i.e. that the diffusion velocity of each element is nil). These models are not time-dependent. Since the diffusion time scales are much smaller in stellar atmospheres than in stellar interiors (for instance see Fig. 3 of LeBlanc 2005), time-dependent diffusion calculations there are thus not numerically feasible for the moment. Such time-dependent calculations would require a large number of time steps and therefore an enormous amount of CPU time. However, the models presented here represent an important step to gauge the potential importance of vertical abundance stratification on the atmospheric structure of certain CP stars.

\section{Theory}

The diffusion velocity of ion i of the trace element A can be approximated by the following equation (Burgers 1960; Vauclair \& Vauclair 1982; Alecian \& Vauclair 1983; Landstreet et al. 1998):

$$
\begin{aligned}
V_{\mathrm{diff}, \mathrm{i}} \approx & D_{\mathrm{i}}\left[-\nabla \ln C_{\mathrm{i}}+\frac{A_{\mathrm{i}} m_{\mathrm{p}} g_{\mathrm{rad}}}{k T}-\right. \\
& \left.\left\{\left(A_{\mathrm{i}}-1\right)+\left(A_{\mathrm{i}}-Z_{\mathrm{i}}\right) f_{\mathrm{p}}\right\} \nabla \ln P\right]
\end{aligned}
$$

where $D_{\mathrm{i}}$ is the diffusion coefficient of the ion under consideration, $A_{\mathrm{i}}$ and $Z_{\mathrm{i}}$ represent its atomic weight and charge, $C_{\mathrm{i}}$ its concentration and $g_{\text {rad }}$ its radiative acceleration. The variables $T$, $P$ and $f_{\mathrm{p}}$ are the local temperature, pressure and HII ionization fraction. The first term found in the bracket is the diffusion that naturally occurs when there exists a concentration gradient relative to depth. This term, which can in some cases vary very rapidly, poses numerical challenges and will be excluded in the numerical simulations presented in this paper. Its potential importance will be discussed later. The second term gives the diffusion related to the momentum transfer to ions from photons following photoexcitation or photoionization. The third term includes gravitational settling and the effect of the electric field. The part of this third term multiplied by $f_{\mathrm{p}}$ is chosen as such because it gives proper asymptotic feature for the electric field in the case where hydrogen is completely neutral and when it is completely ionized (Landstreet et al. 1998). Other terms, such as thermal diffusion (which is negligible in the atmosphere) and exchange terms among the species, have been neglected here.

The most important ingredient in the diffusion equation is the radiative acceleration. For an accurate determination of this term, proper atomic data of all the elements present in stars must be known along with the radiative flux (for instance, see Eq. (1) of Hui-Bon-Hoa et al. 2002). The radiative acceleration is calculated by integrating the monochromatic opacity of each ion times the flux over the whole spectrum. The opacity must then be sampled (e.g. LeBlanc et al. 2000) on a sufficiently refined frequency grid for this integral to be precise. In the calculations presented here, two different grids with up to nearly a quarter of a million frequency points were used. It should be noted that $g_{\text {rad }}$ has a non-linear dependence on the abundance of the element under consideration (e.g. Alecian \& LeBlanc 2000) and also depends on the abundances of the other elements through the radiation field.

The presence of a magnetic field can also modify the radiative accelerations via the Zeeman effect (Alecian \& Stift 2004). The diffusion of charged ions is also affected by magnetic field lines (e.g. Hui-Bon-Hoa et al. 1996) through the diffusion coefficient. Only this last magnetic effect will be considered here.

As previously mentioned, our models do not take into account time-dependent diffusion such as in the evolutionary models of Turcotte et al. (1998) and Michaud et al. (2004) for example. The abundances calculated here approximate the maximum values that can be supported by the radiation field at each point of the atmosphere. Phenomena occurring below the atmosphere could hinder this from happening in real stars and is a source of uncertainty in our models. Other effects such as massloss could also play a role. However, the models presented here are among the first attempts to include abundance stratification due to atomic diffusion in stellar atmospheres and to evaluate its potential importance on the atmospheric structure. This is an important step towards a better understanding of the atmospheres of certain stars that have hydrodynamically stable atmospheres where diffusion could occur.

\section{Models}

The models presented here are calculated with a modified version of version 11 of the PHOENIX code (e.g. Hauschildt et al. 1999), in LTE mode, previously used in Hui-Bon-Hoa et al. (2000). A total of 39 elements (H-Ga, Kr-Nb, Ba and La) are included in this code.

Radiative accelerations for both bound-bound and boundfree transitions were included here. For example, the radiative acceleration (assuming no redistribution of the momentum among the ions, see discussion below) on an ion $A_{\mathrm{i}}$ due to boundbound transitions is

$g_{\mathrm{rad}}^{\mathrm{bb}}\left(A_{\mathrm{i}}\right)=\frac{4 \pi}{c} \frac{1}{X_{A_{\mathrm{i}}}} \int_{0}^{\infty} \kappa_{\lambda}^{\mathrm{bb}}\left(A_{\mathrm{i}}\right) H_{\lambda} \mathrm{d} \lambda$

where $\kappa_{\lambda}^{\mathrm{bb}}\left(A_{\mathrm{i}}\right)$ is the opacity due to bound-bound transitions and $H_{\lambda}$ is the Eddington flux. A similar equation can be written for 
bound-free transitions. However, for bound-free transitions, the momentum gained by ions of charge $Z$, comes from the absorption of photons by the ions with charge $Z-1$. More details concerning the theoretical aspects surrounding the evaluation of radiative accelerations can be found in Hui-Bon-Hoa et al. (2002), Alecian \& LeBlanc (2000) and Gonzalez et al. (1995). The line opacities used here are calculated using Kurucz' (1994) linelist. The bound-free absorption coefficients are assumed to be hydrogenic (i.e. proportional to $v^{-3}$, see Hui-Bon-Hoa et al. 2002, for more details) and it is assumed that no momentum is taken away by the ejected electron.

As mentioned previously, radiative accelerations are obtained by integration on frequency grids that properly sample the opacity spectrum. For the $8000 \mathrm{~K}$ model, the grid used in this paper is the following:

$$
\begin{aligned}
& \Delta \lambda=0.5 \AA \text { for } 1 \AA \leq \lambda<350 \AA \\
& \Delta \lambda=0.05 \AA \text { for } \quad 350 \AA \leq \lambda<10^{4} \AA \\
& \Delta \lambda=2 \AA \text { for } 10^{4} \AA \leq \lambda<10^{5} \AA \\
& \Delta \lambda=10^{4} \AA \text { for } 10^{5} \AA \leq \lambda<10^{6} \AA \\
& \Delta \lambda=10^{5} \AA \text { for } 10^{6} \AA \leq \lambda \leq 10^{7} \AA
\end{aligned}
$$

This grid is chosen so that its maximal resolution $\Delta \lambda=0.05 \AA$ is sufficient to properly sample the spectral region where most of the atomic lines are present. This resolution is such that it is smaller than typical line widths. Even though the radiative acceleration of each atomic line is not precisely obtained, on average, the overall acceleration for the given species is sufficiently precise. However, it should be mentionned that opacity sampling cannot precisely evaluate radiative accelerations of species with a small number of lines. Only detailed calculations with a large number of frequency points within the lines can achieve precise results in this particular case. This drawback is not of critical importance for the study of the effect of stratification on the atmospheric structure since such species will generally not be stongly supported by radiation and will not significantly affect the overall opacity spectrum. The grid used for the $12000 \mathrm{~K}$ is twice as coarse as the grid described above (see below for more details).

The first improvement brought to the code is the implementation of a new iterative diffusion velocity convergence algorithm. This algorithm is used to calculate equilibrium abundances of chemical elements as a function of depth in the atmosphere and it aims to find the abundance in each layer of the model needed to give a nil diffusion velocity for each element considered. As will be shown below, this can create highly non-uniform vertical distributions.

The algorithm employed uses an iterative process. In selfconsistent model atmospheres, the atmospheric structure has to be adjusted each time the abundances are modified. Therefore, abundance corrections are followed by temperature corrections. In turn, the changes in the physical structure lead to an imbalance of the forces acting on the chemical elements. The diffusion velocities become non-zero, and the abundances have to be corrected again in order to compensate for this imbalance.

In our calculations, the abundance correction applied for a given element is proportional to its diffusion velocity. The atmosphere is divided into 50 layers, and $V_{\text {diff }}$ is calculated in each layer and is then used to compute abundance corrections in each of these layers. The abundance is multiplied by the correction factor $1+\frac{V_{\text {diff }}}{K}$, where $\mathrm{K}$ is a constant chosen so that $\left|\frac{V_{\text {diff }}}{K}\right|<1$.

If $V_{\text {diff }}$ is positive, the abundance is increased and it is decreased if $V_{\text {diff }}$ is negative, the reason being that $g_{\text {rad }}$ increases when the abundance decreases due to the desaturation of the spectral lines. Abundance corrections should be small enough so that the algorithm remains robust. The radiative transfer equation is solved, for all wavelengths, after every abundance or temperature correction.

We also improved the diffusion coefficients used in the code, since we now include the effect of interactions betweens the ions and neutral hydrogen, in addition to their interaction with protons (e.g. Budaj \& Dworetsky 2002; Landstreet et al. 1998). The effect of horizontal magnetic fields on diffusing ions (Alecian \& Vauclair 1981) was also added to the present code (see Sect. 4.1.3).

When an ion of a given element absorbs a photon, the acquired momentum can be spent while the ion is in the same ionization state or, depending on what happens to the ion, it can be spent in another ionization state. For instance, the initial ion can be ionized immediately following the absorption of the photon mentioned previously, and the momentum will then be given to the newly ionized ion. Similarly, if the absorbing ion recombines soon after this absorption, the momentum will then be spent while the ion is in the newer less ionized state. This process of redistribution of momentum among the ions can strongy affect radiative acceleration since more highly ionized species have less mobility or lower diffusion coefficients (Montmerle \& Michaud 1976; Gonzalez et al. 1995). Redistribution depends on the collisional, ionization and recombination rates and it is therefore very difficult to estimate with precision. This can lead to relatively large uncertainties for radiative accelerations, especially in the atmospheres of stars where redistribution can be very important due to the presence of neutral ions. In the calculations presented here, a redistribution scheme proposed by Montmerle \& Michaud (1976) was used, as described in Hui-Bon-Hoa et al. (1996). More details concerning $g_{\text {rad }}$ calculations can be found in Hui-Bon-Hoa et al. (2002). Results will also be shown while using other approximations related to redistribution.

For the hotter models, the total radiative acceleration on matter can in some cases become greater than gravity. This is due to the fact that we are calculating the approximate maximum amount of each species that can be supported by radiative diffusion. Some elements can then become very overabundant. In more realistic time-dependent diffusion models, such large amounts of matter might not have time to diffuse to the outer regions. To eliminate this problem and in an attempt to avoid conditions not observed in the type of stars under consideration here, we impose that the maximum abundance permitted for each species is 1000 times its solar value. This is a reasonable value, since the chemical elements in our set are not observed at such high overabundances in most CP stars.

The diffusion equation assumes that the diffusing species is a trace element (i.e., the test particle approximation). However, since we calculate here the maximum abundance that can be supported by radiative diffusion, the abundance of certain elements at certain depths can become very large and violate the test particle approximation. In order to avoid such theoretical inconsistencies, we set a maximum abundance (as compared to the total number of atoms) of 0.005 or one part per 200.

Also, at some depths, certain elements are simply not supported by their radiative force that is always weaker than gravity. In this context, our models tend towards a zero abundance for these elements at the concerned depths. In reality, other dynamical processes such as mass-loss would lead to a non nil abundance for these elements. To avoid this inconsistency, we imposed a minimum abundance for such cases to a value of 0.001 times the solar abundance of the elements in question. 
The minimal and maximal values for the abundance imposed and discussed above are arbitrary. However, there are sound reasons related to the limitations of our models that lead us to impose these abundance limits.

\section{Results}

For the self-consistent model atmospheres presented below (Sect. 4.2), the abundance of each element, except for $\mathrm{H}$ which acts as the buffer gas, is calculated by imposing that its diffusion velocity is nil everywhere, when possible, while the atmospheric structure is calculated with the new abundance profiles. The number fraction of $\mathrm{H}$ is normalized such that it is equal to one minus the sum of the number fractions of the other elements. But first, some results will be shown while neglecting the correction to the structure.

\subsection{Results with no temperature correction}

\subsubsection{Convergence}

In this section, we examine how the diffusion velocities and the abundance profiles converge while using chemically homogeneous model atmospheres (i.e., no temperature correction was undertaken here, the underlying $T-\tau$ relation was that of a chemically homogeneous model for which the hydrostatic equilibrium equation was solved for each iteration). Since $\mathrm{Fe}$ is an important element in most CP stars, results featuring this element will be shown for illustrative purposes. Figure 1 shows the convergence of the diffusion velocity for $\mathrm{Fe}$ in models with $T_{\text {eff }}=8000 \mathrm{~K}$ and $T_{\text {eff }}=12000 \mathrm{~K}$ (both with $\log g=4.0$ ). For the $8000 \mathrm{~K}$ model we used an atmosphere model with solar abundances and began the iteration process with these abundances. For the $12000 \mathrm{~K}$ model we chose to use a metallicity of -1.5 dex the solar value. These last abundances were chosen since they are typical of globular clusters where BHB stars are mostly found (e.g. Grundahl et al. 1999).

We clearly see that the convergence algorithm used strongly diminishes the diffusion velocity. In the examples shown here, the diffusion velocity is diminished by two to six orders of magnitudes as compared to its initial value in the atmosphere. In these calculations, the Montmerle \& Michaud (1976) redistribution scheme was employed. We also note that the cooler of these two models converges faster than the hotter one. In order to better illustrate the convergence scheme, no upper or lower limits were imposed on the abundances (see discussion in previous section) for the results shown in Figs. 1 and 2.

It should be noted that the frequency grid used for the hotter model has half the resolution of the one used in the cooler model shown which comprises of nearly of a quarter million frequency points. This was necessary to limit the CPU time needed for the convergence of the self-consistent models with $T_{\text {eff }}=12000 \mathrm{~K}$. Since the lines are wider in the hotter models, this compromise still gives relatively accurate radiative accelerations. For example, the error due to the decrease of the frequency grid refinement on the supported $\mathrm{Fe}$ abundance is less that 0.2 dex in most regions of the atmosphere.

Figure 2 illustrates the calculated abundances for iron at various abundance correction iterations as we seek to reach equilibrium (i.e. $V_{\text {diff }}=0$ ) for the same two models used above. We observe very different abundance profiles in these two models. However, in both models we see a large overabundance bump (as compared to solar values, at $i=1$ ) due to the increase of the opacity of iron with depth. In the $8000 \mathrm{~K}$ model, we see
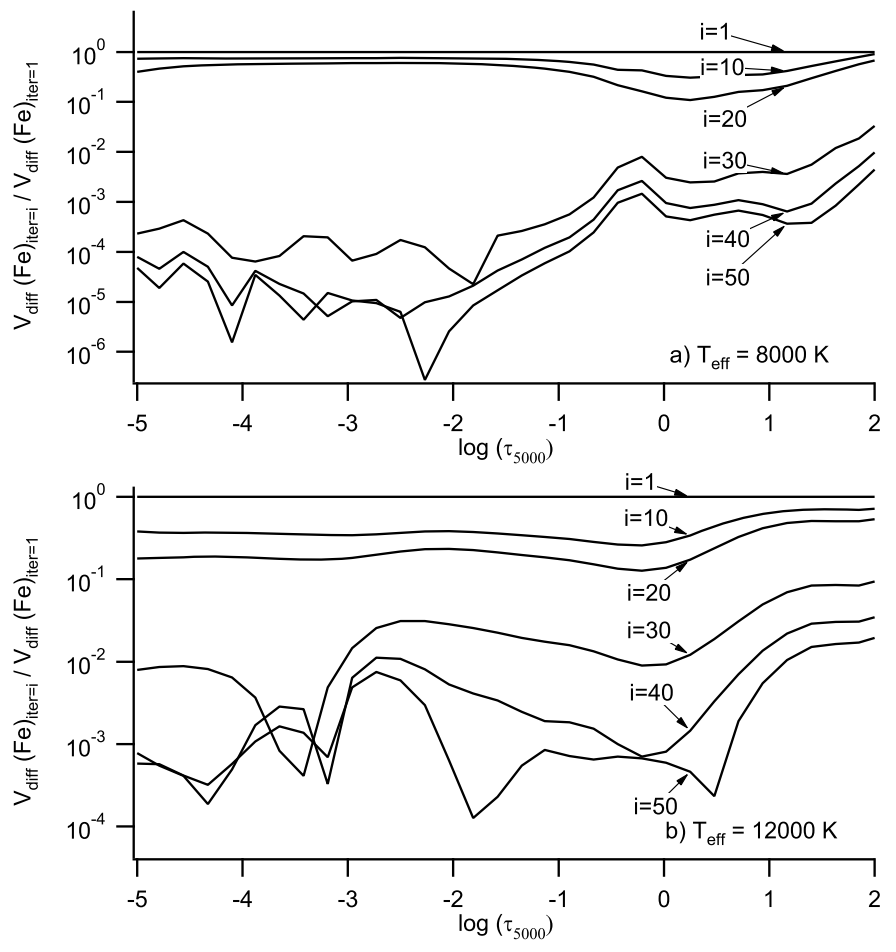

Fig. 1. The ratio of the diffusion velocity of $\mathrm{Fe}$ at various iterations $i$ as compared to that of the first iteration in models with a) $T_{\text {eff }}=8000 \mathrm{~K}$ and b) $T_{\text {eff }}=12000 \mathrm{~K}$ (both with $\log g=4.0$ ). These calculations are performed using a chemically homogeneous model atmosphere with the chosen initial abundances.

that in the exterior regions $\left(\log \left(\tau_{5000}\right) \sim-3\right)$ Fe is not strongly supported by the radiative field. However, in a real star, the Fe abundance might not be as small in this region since mass loss could for example limit this abundance decrease. This is one of the reasons that for the results shown in the rest of this paper, the abundance limits discussed in Sect. 3 are imposed.

\subsubsection{The effect of redistribution of the momentum}

Figure 3 shows the predicted abundance stratification of iron for various redistribution schemes. Four curves are shown. A curve is shown where we impose no redistribution among the ions. In other words, we suppose that all of the acquired momentum is spent in the initial ionic stage. This may be regarded as an upper limit for the radiative accelerations, since recombination rates are generally much less important than ionization rates (e.g. Gonzalez et al. 1995). A second curve is shown using the method described in Montmerle \& Michaud (1976) which depends on the relative values of the collisional, ionization and recombination rates. A third curve shows a redistribution curve where we suppose that all of the momentum is transferred to the next higher ionization state. This may be considered as a lower limit for the radiative accelerations. Finally a curve assuming that all of the ions possess the same diffusion coefficient is shown. For this case, redistribution does not affect the results since all ions have the same mobility.

For the $T_{\text {eff }}=8000 \mathrm{~K}$ model atmosphere, we can notice that the redistribution scheme of Montmerle \& Michaud (1976) is quite close to that assuming no redistribution. In the $T_{\text {eff }}=$ $12000 \mathrm{~K}$ model, the Fe abundance profile strongly depends on redistribution. Comparisons to observed abundance profiles such as those of Khalack et al. (2007 and 2008) could shed light on the 

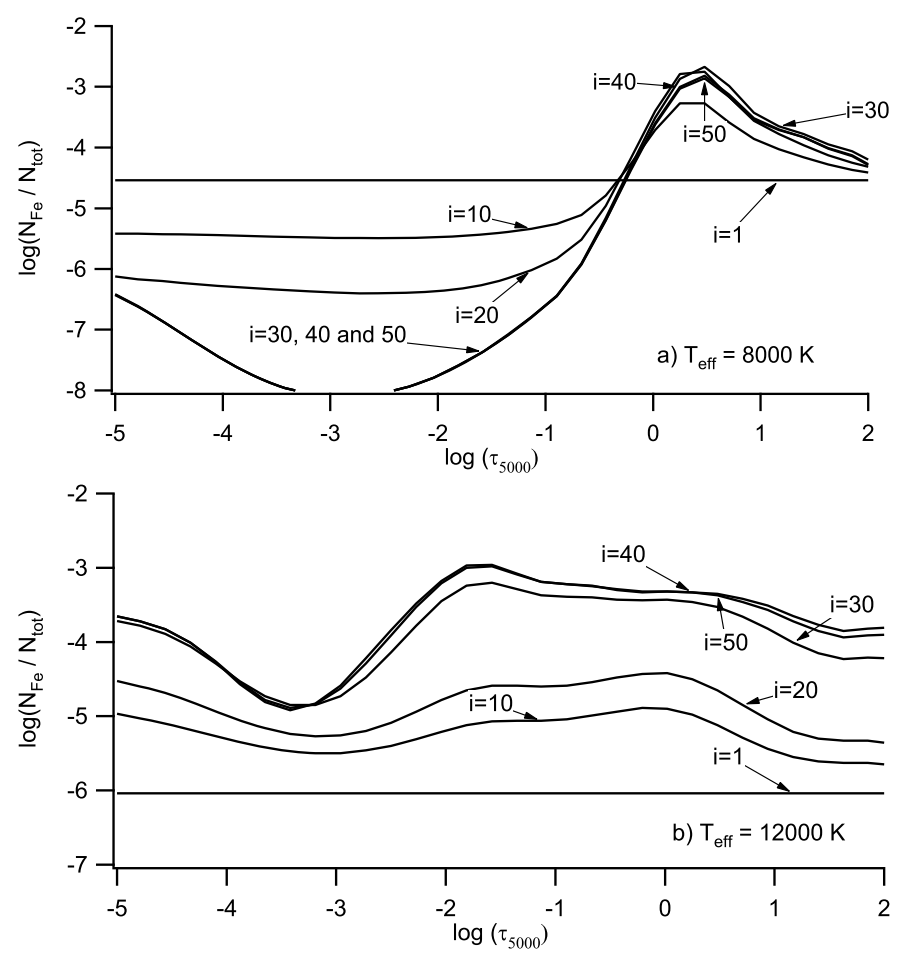

Fig. 2. The supported abundance of $\mathrm{Fe}$ at various iterations $i$ as compared to that of the first iteration in models with a) $T_{\text {eff }}=8000 \mathrm{~K}$; and b) $T_{\text {eff }}=12000 \mathrm{~K}$ (both with $\log g=4.0$ ). These calculations are performed using a chemically homogeneous model atmosphere with the chosen initial abundances.

level of redistribution taking place in such stars. In both models and as expected, the abundances supported by diffusion when assuming that all of the momentum is redistributed to the next ion is much lower than the other three cases. The position and the intensity of the main abundance gradient found for Fe strongly depends on redistribution.

\subsubsection{The presence of magnetic fields}

Since Ap stars may have strong magnetic fields, it is essential to gauge the effect of these fields on the predicted abundance stratifications. Magnetic fields can affect elemental stratification through two effects. The first, that will be neglected here, is the Zeeman effect (Alecian \& Stift 2004). Generally, Zeeman splitting will desaturate atomic lines and therefore increase $g_{\text {rad }}$. A second way that magnetic fields can play a role is related to the interaction of diffusing ions with these fields. The horizontal component of the magnetic field can hinder the vertical diffusion of the ions (e.g. Hui-Bon-Hoa et al. 1996).

These models predict that Fe is supported at much shallower regions when horizontal magnetic fields are present (see Fig. 4). The magnetic field decreases the effective diffusion coefficient of charge ions. This increases the relative importance of FeI and leads, in this case, to a larger radiative acceleration. Deeper in the atmosphere, the presence a magnetic field is less important since collisional frequencies become larger than the Larmor frequency. Similar results were found by Alecian \& Stift (2007).

\subsection{Self-consistent models with stratification}

In this section, we will investigate the effect of vertical stratification in models for which the structure is calculated while
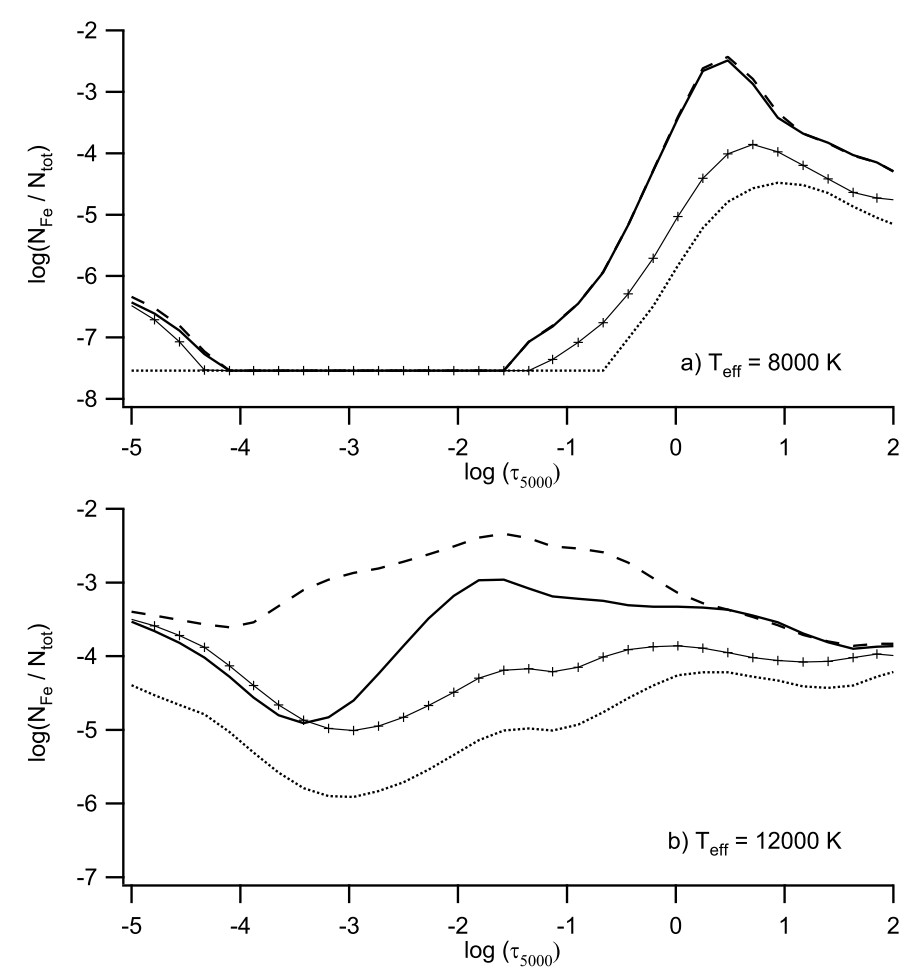

Fig. 3. The supported abundance of $\mathrm{Fe}$ for various redistributions in models with a) $T_{\text {eff }}=8000 \mathrm{~K}$ with $\log g=4.0$; and b) $T_{\text {eff }}=12000 \mathrm{~K}$ with $\log g=4.0$. The dashed curve assumes no redistribution, the solid curve assumes a redistribution calculated as in Montmerle \& Michaud (1976) and the dotted curve assumes that all of the momentum is redistributed to the next higher ionization state. The solid line with crosses represents calculations assuming that all of the ions have similar diffusion coefficients. These calculations are performed using a chemically homogeneous model atmosphere with the chosen initial abundances.

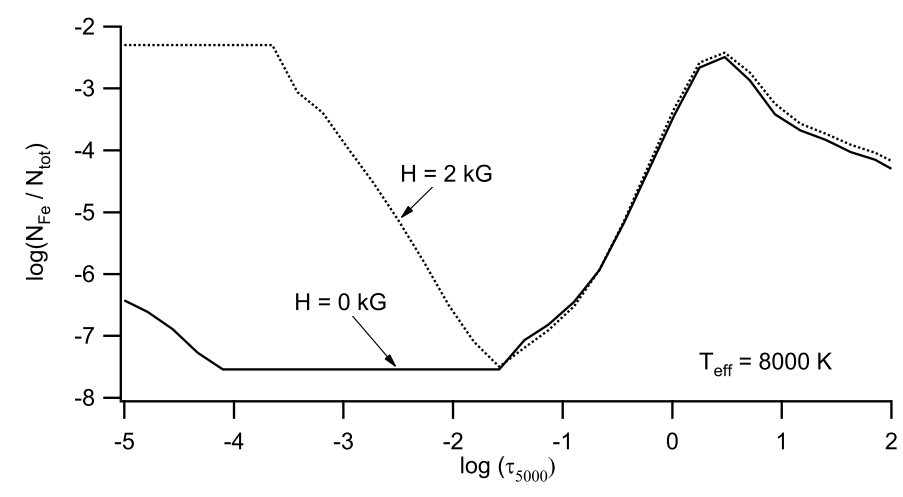

Fig. 4. The supported abundance of Fe in a model with $T_{\text {eff }}=8000 \mathrm{~K}$ and $\log g=4.0$ for two values for the horizontal magnetic field: $H=$ 0 and $2 \mathrm{kG}$. The Montmerle \& Michaud (1976) redistribution scheme was used for both curves and these calculations are performed using a chemically homogeneous model atmosphere with the chosen initial abundances.

taking into account these abundance gradients. Both the changes in structure and abundances can modifiy the radiative accelerations of the elements. The temperature alterations change the opacity via the modification brought about to the atomic populations. Meanwhile the change in abundances with depth, modifies the line blending and thus the saturation effects. This can lead to significant changes in radiative accelerations and therefore also to the amount of a given species that can be supported by radiative diffusion. We will begin by evaluating the changes in the 

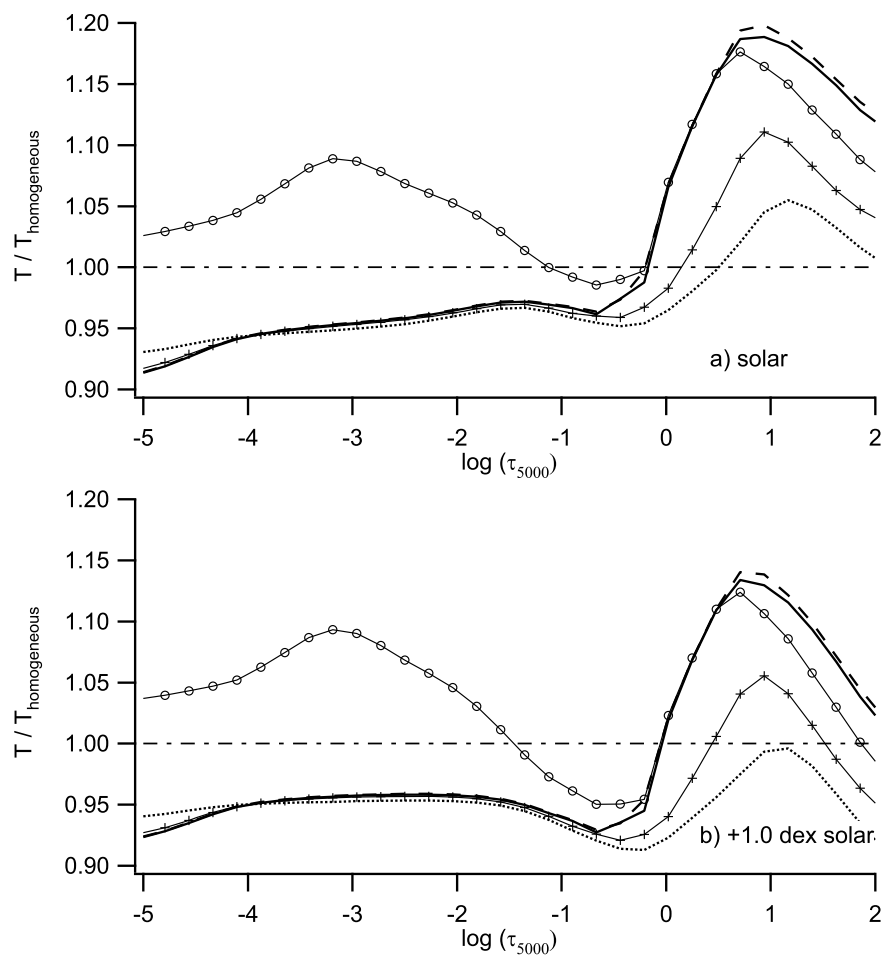

Fig. 5. The ratio of the temperature of various models including stratification (with $T_{\text {eff }}=8000 \mathrm{~K}$ and $\log g=4.0$ ) as a function of depth to that of a chemically homogeneous model with a) solar composition; and b) metal abundances at 10 times solar values. The various curves shown are those defined in Fig. 3, while the solid curve with circles is a model assuming the Montmerle \& Michaud (1976) redistribution with a horizontal magnetic field of $2 \mathrm{kG}$. The dashed-dotted curve represents the value of unity.

temperature profile due to elemental stratification for a $8000 \mathrm{~K}$ model.

Figure 5 shows the ratio of the local temperature of selfconsistent models with various assumptions as compared to chemically homogeneous models assuming solar abundances and a metallicity enhanced by +1 dex. These two cases were chosen to gauge the relative effect of simply choosing to use a chemically homogeneous model with differing metallicity as compared to a model with vertical abundance stratification. Also, since Ap stars generally have enriched abundances, it is instructive to also compare the temperature profile of the self-consistent models to that of a chemically homogeneous model with a metallic abundance of 10 times the solar value.

In the deeper regions $\left(\log \left(\tau_{5000}\right) \sim 1\right)$ of the models, the modifications brought about to the temperature by stratification can be quite large (up to 20\%) and are strongly dependent on redistribution. As expected, the ratio showed for the metallically enriched model is smaller than the one for the solar homogeneous model since many elements in these deeper regions are well supported by radiative diffusion. However, the temperature profile of the self-consistent models are very different from both chemically homogeneous models used here.

Figure 6 shows the Fe stratification profile in the $8000 \mathrm{~K}$ model. For all cases shown, we find a large increase of the Fe abundance within the region $-1 \leq \log \left(\tau_{5000}\right) \leq 0$.

Figure 7 shows that stratification of the elements leads to large variations of temperature in most of the cases shown for the $12000 \mathrm{~K}$ models. Since the chemically homogeneous model with a metallicity of 10 times solar was chosen to compare our

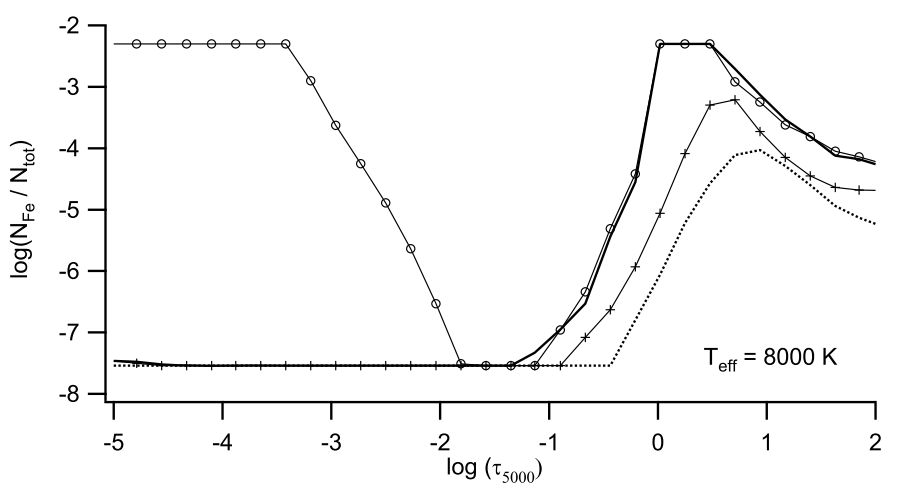

Fig. 6. The iron abundance as a function of optical depth for models with $T_{\text {eff }}=8000 \mathrm{~K}$ and $\log g=4.0$. The various curves shown are those defined in Fig. 5. The case assuming no redistribution is not shown since it is almost indistinguishable from the solid curve.
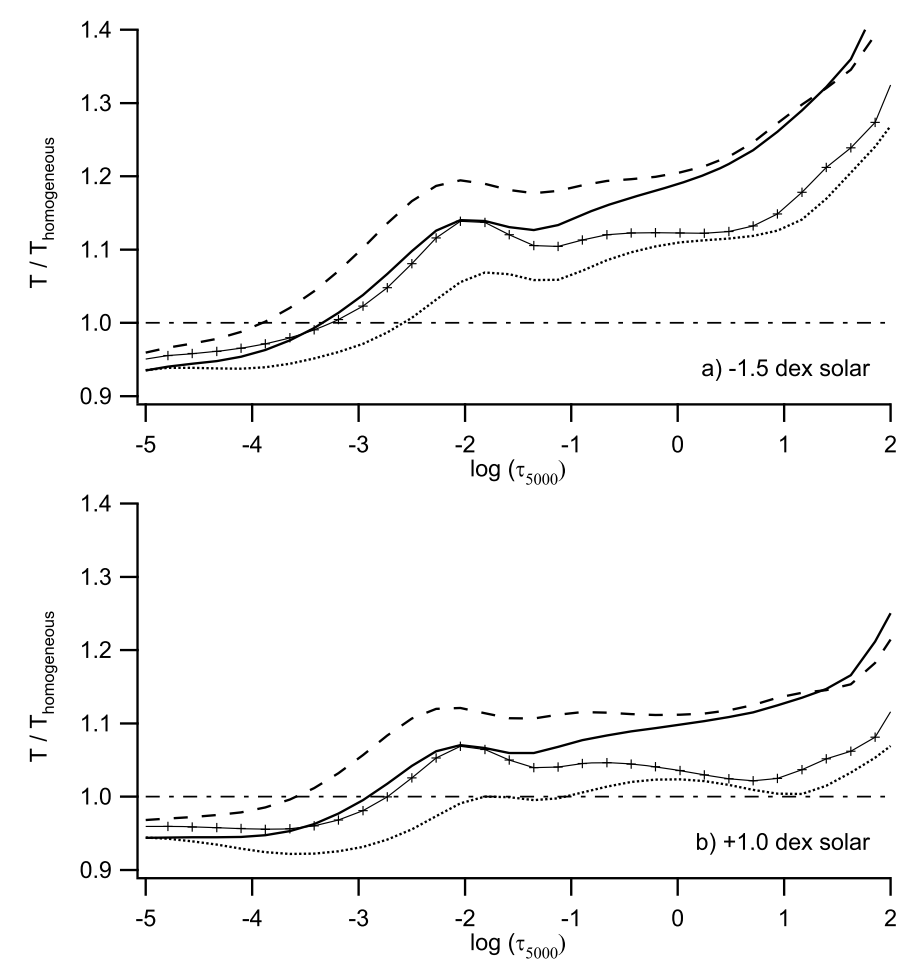

Fig. 7. The ratio of the temperature of various models including stratification (with $T_{\text {eff }}=12000 \mathrm{~K}$ and $\log g=4.0$ ) as a function of depth to that of a chemically homogeneous model with a) metals at $-1.5 \mathrm{dex}$ times solar values; and b) metal abundances at 10 times solar values. The various curves shown are those defined in Fig. 5.

results to typical of BHB stars, the ratio that is of most interest is the one shown in Fig. 7b, the reason being that most metals are generally observed to be overabundant in the atmospheres of these stars (e.g. Grundahl et al. 1999). When using the redistribution scheme of Montmerle \& Michaud (1976), the temperature is increased by approximately $10 \%$ or more for $\log \left(\tau_{5000}\right) \geq-2$ as compared to the chemically homogeneous model with a metallicity of 10 times solar.

Figure 8 shows that the Fe stratification profile depends strongly on the redistribution of momentum among the ions. When assuming no redistribution, the $\mathrm{Fe}$ abundance reaches the maximum allowed abundance of one part per 200 particles for $\log \left(\tau_{5000}\right) \leq-2$. The structural changes due to stratification also decreases and displaces the $\mathrm{Fe}$ abundance gradient seen in the 


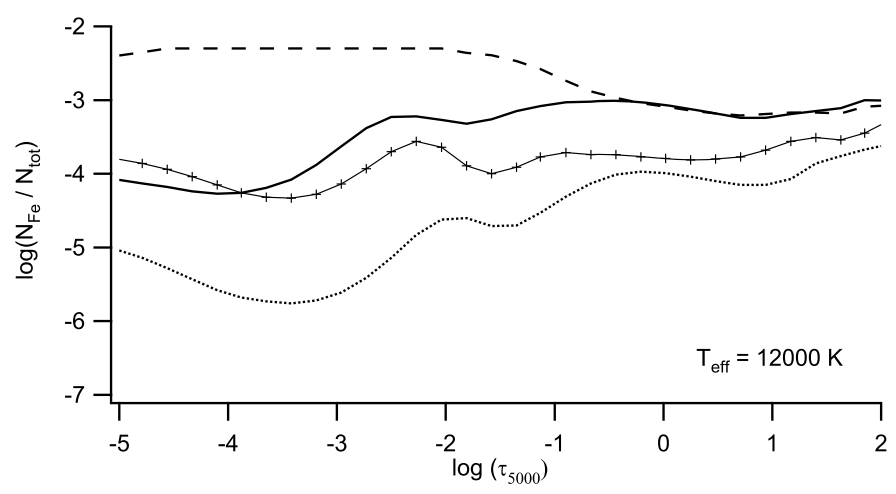

Fig. 8. The iron abundance as a function of optical depth for models with $T_{\text {eff }}=12000 \mathrm{~K}$ and $\log g=4.0$. The various curves shown are those defined in Fig. 3.
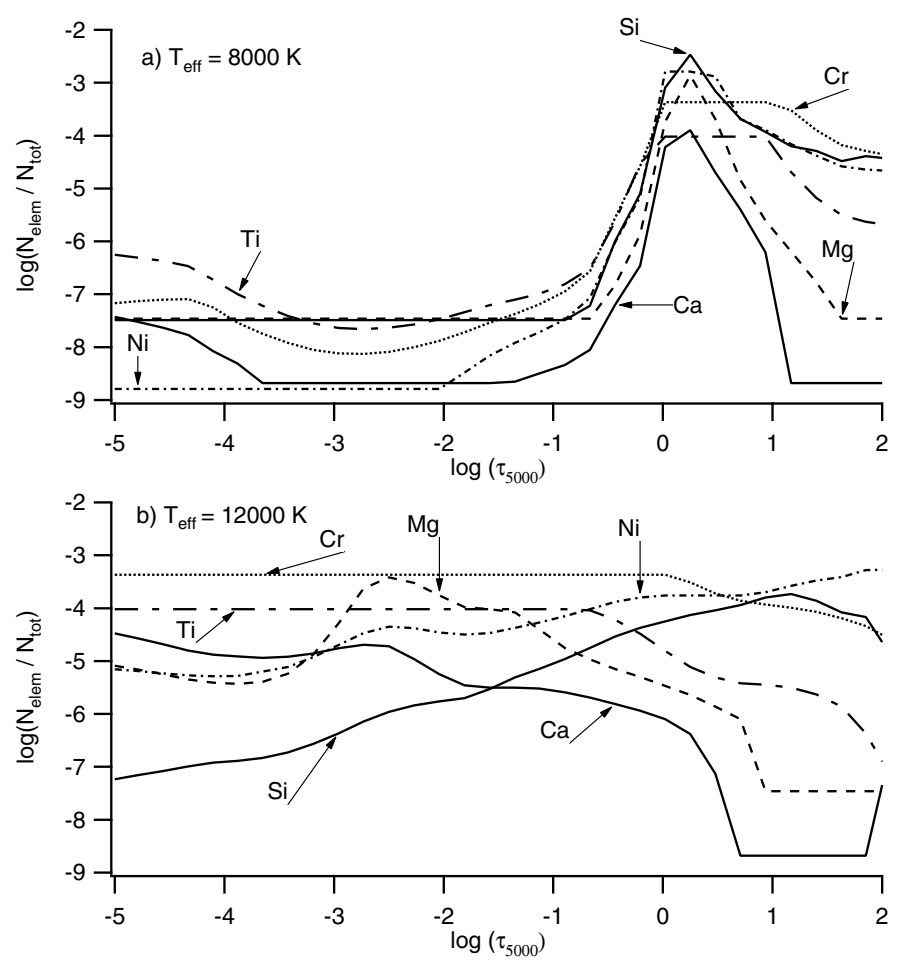

Fig. 9. The abundance of the elements $\mathrm{Mg}, \mathrm{Si}, \mathrm{Ca}, \mathrm{Ti}, \mathrm{Cr}$ and $\mathrm{Ni}$ as a function of optical depth for models with a) $T_{\text {eff }}=8000 \mathrm{~K}$ and b) $T_{\text {eff }}=12000 \mathrm{~K}$ (both for $\log g=4.0$ ) assuming the Montmerle \& Michaud (1976) redistribution.

region where $-3.5 \leq \log \left(\tau_{5000}\right) \leq-2$ as compared to the results shown in Fig. 3.

Figure 9 shows representative results for the stratification found for a few elements of interest in the $8000 \mathrm{~K}$ and $12000 \mathrm{~K}$ models. The general form of the stratification profiles in the $8000 \mathrm{~K}$ model are very similar from one element to another, while those in the $12000 \mathrm{~K}$ model vary greatly. The relative abundances of these elements that are supported in the atmosphere of these two models are also considerably different.

\section{Discussion and conclusion}

In this paper, we presented an improved version of model atmospheres while including vertical chemical stratification of Hui-Bon-Hoa et al. (2000). The first generation of our models were successful in qualitatively reproducing the photometric jumps and gaps observed for BHB stars. They were thereafter applied to Ap stars (Ryabchikova et al. 2003; LeBlanc 2003) but without including any magnetic effects.

The temperature change due to stratification in the line forming region of the $8000 \mathrm{~K}$ model shown in Fig. 5 is relatively small except for the case with a magnetic field. In the line forming region, the maximum temperature increase in the model with a magnetic field of $2 \mathrm{kG}$ is on the order of $500 \mathrm{~K}$ and occurs at $\log \left(\tau_{5000}\right) \sim-3$. This temperature increase, which depends on the magnetic field strength, might be able to explain the well-known core-wing anomaly of hydrogen Balmer lines observed in cool Ap stars. This anomaly is defined by broad wings and relatively narrow cores (Wegner 1976; Cowley et al. 2001). Kochukhov et al. (2002) showed that an empirical increase of the temperature by 500 to $1000 \mathrm{~K}$ in the atmospheric layers with $-4 \leq \log \left(\tau_{5000}\right) \leq-1$ can reproduce the core-wing anomaly. The theoretical results shown in Fig. 5 seem to confirm that such a temperature increase is possible in Ap stars. More detailed calculations will be undertaken in the near future to verify if our theoretical models can explain this well-known anomaly.

Wade et al. (2001) found that the lines of $\mathrm{Ca}, \mathrm{Fe}$ and $\mathrm{Cr}$ in the Ap star $\beta$ CrB could be fitted better by using an empirical twozone abundance profile with a sharp transition between these two abundance zones than a chemically homogeneous abundance. In these simulations, the underlying atmospheric model was calculated with a vertically homogeneous composition. Such an empirical stratification profile has also been applied to other Ap stars (e.g. Ryabchikova et al. 2005; Ryabchikova 2005). A two-zone abundance profile has also been employed for the study of possible stratification in HgMn stars (Smith 1995). The abundance jump seen in Fig. 6 is qualitatively consistent to those found in the studies of Ap stars just mentioned. The models shown here assume that the atmospheres of Ap stars are completely stable. However, even though no sign of convection is observed in the atmospheres of these stars, it cannot be excluded that a slow mixing zone could persist. LeBlanc (2005) showed that this may lead to a flattening of the abundance bump seen approximately between $\log \left(\tau_{5000}\right)=-0.5$ and 2 for the $8000 \mathrm{~K}$ model.

Since BHB stars are non-magnetic, the models presented here should better reproduce certain observational anomalies observed for these stars. Khalack et al. (2008) detected Fe stratification in the atmosphere of three BHB stars. They found that when assuming zero microturbulent velocity the Fe abundance first decreases and then increases as a function of optical depth in the line forming region. This same feature can be seen in Figs. $2 b$ and 8 where the minimum of $\mathrm{Fe}$ abundance is respectively found near $\log \left(\tau_{5000}\right) \sim-3$ and -4 . However, this same study showed that the presence of weak microturbulence can erase this upturn of the Fe abundance observed at low optical depths. The stability of the atmosphere is therefore critical for this case. In future studies, we plan to apply our models to these stars and especially to recent detections of vertical abundance stratification found by Khalack et al. (2007 and 2008).

The models presented here have several drawbacks. As mentioned, they are not time-dependent. Also, since these are one dimensional the complex magnetic field configurations of Ap stars cannot be taken into account. However, simulations using models with various values for the magnetic field could be used to simulate the horizontal abundance gradients observed at their surface. However, the Zeeman effect which is not included in our models probably plays an important role in such phenomena. Uncertainties also remain regarding the effectiveness of redistribution. The models presented here also have limitations 
pertaining to incomplete atomic data and neglect of heavy elements in the present version of PHOENIX code. This is especially important for Ap stars.

Several physical phenomena were neglected here. First, the term related to the abundance gradient was not included. Monin \& LeBlanc (2007) have included this term exclusively for iron while using an underlying chemically homogeneous model atmosphere and they found the abundance jump of Fe in Ap stars can be shifted to lower optical depths (by less than 0.5 dex). The general shape of the abundance profile remains similar, but the abundance at some of the points in the part of the profile that has a strong gradient can be increased significantly. Other effects such as mass loss, Zeeman splitting and the possible presence of slow mixing due to convection (LeBlanc 2005) were also neglected here. However, the models presented here are an important step to better understanding the importance of vertical abundance stratification in stellar atmospheres.

Acknowledgements. This research was partially funded by NSERC and La Faculté des Études Supérieures et de la Recherche de l'Université de Moncton. Computational resources were provided by the Réseau québécois de calcul de haute performance (RQCHP). One of us (FL) is grateful for a visiting professorship position at l'Université de Toulouse.

\section{References}

Alecian, G., \& LeBlanc, F. 2000, MNRAS, 319, 677

Alecian, G., \& Stift, M. J. 2004, A\&A, 416, 703

Alecian, G., \& Stift, M. J. 2007, A\&A, 475, 659

Alecian, G., \& Vauclair, S. 1981, A\&A, 101, 16

Alecian, G., \& Vauclair, S. 1983, Fund. Cosm. Phys., 8, 369

Babel, J. 1994, A\&A, 283, 189

Budaj, J., \& Dworetski, M. M. 2002, MNRAS, 377, 1340

Bagnulo, S., Wade, G. A., Donati, J.-F., et al. 2001, A\&A, 369, 889

Burgers, J. M. 1960, in Plasma Dynimics, ed. F. H. Clauser (Reading: AddisonWesley), 119

Cowley, C. R., Hubrig, S., Ryabchikova, T. A., et al. 2001, A\&A, 367, 939

Dreizler, S., \& Wolff, B. 1999, A\&A, 348, 189

Gonzalez, J.-F., LeBlanc, F., Artru, M.-C., \& Michaud, G. 1995, A\&A, 297, 223

Grundahl, F., Catelan, M., Landsman, W. B., Stetson, P. B. \& Andersen, M. I. 1999, ApJ, 524, 242

Hauschildt, P. H., Allard, F., \& Baron, E. 1999, ApJ, 512, 377

Hui-Bon-Hoa, A., Alecian, G., \& Artru, M.-C. 1996, A\&A, 313, 624
Hui-Bon-Hoa, A., LeBlanc, F., \& Hauschildt, P. H. 2000, ApJ, 535, L43

Hui-Bon-Hoa, A., LeBlanc, F., Hauschildt, P. H., \& Baron, E. 2002, A\&A, 381, 197

Khalack, V. R., LeBlanc, F., Bohlender, D., Wade, G. A., \& Behr, B. B. 2007, A\&A, 466, 667

Khalack, V. R., LeBlanc, F., Behr, B. B., Wade, G. A., \& Bohlender, D. 2008, A\&A, 477, 641

Kochukhov, O., Bagnulo, S., \& Barklem, P. S. 2002, ApJ, 578, L75

Kochukhov, O., Tsymbal, V., Ryabchikova, T., Makaganyk, V., \& Bagnulo, S. 2006, A\&A, 460, 831

Kurucz, R. L. 1994, Atomic Data for Opacity Calculations (Kurucz CD-ROM No. 1)

Landstreet, J. D., Dolez, N., \& Vauclair, S. 1998, A\&A, 333, 977

LeBlanc, F. 2003, in International Conference on Magnetic Fields in O, B and A Stars, ed. L. A. Balona, H. F. Henrichs, \& R. Medupe, ASP Conf. Ser., 305, 206

LeBlanc, F. 2005, in Element Stratification in Stars, 40 Years of Atomic Diffusion, ed. G. Alecian, O. Richard, \& S. Vauclair, EAS PS, 17, 73

LeBlanc, F., \& Monin, D. 2004, in The A-star Puzzle, ed. J. Zverko, J. Ziznovsky, S. J. Adelman, \& W. W. Weiss, IAU Symp., 224, 193

LeBlanc, F., Michaud, G., \& Richer, J. 2000, ApJ, 538, 876

Michaud, G. 1970, ApJ, 160, 641

Michaud, G., Richard, O., Richer, J., \& VandenBerg, D. A. 2004, ApJ, 606, 452

Michaud, G., Richer, J., \& Richard, O. 2008, ApJ, 675, 1223

Monin, D., \& LeBlanc, F. 2007, in Physics of Magnetic Stars, 360

Montmerle, T., \& Michaud, G. 1976, ApJS, 31, 489

Ryabchikova, T. 2005, in Element Stratification in Stars: 40 Years of Atomic Diffusion, ed. G. Alecian, O. Richard, \& S. Vauclair, EAS PS, 17, 253

Ryabchikova, T., Piskunov, N., Khochukhov, O., et al. 2002, A\&A, 384, 545

Ryabchikova, T., Wade, G., \& LeBlanc, F. 2003, in Modelling of Stellar Atmospheres, ed. N. Piskunov, W. W. Weiss, \& D. F. Gray, IAU Symp., 210, 301

Ryabchikova, T., Leone, F., \& Kochukhov, O. 2005, A\&A, 438, 973

Ryabchikova, T., Khochukhov, O., \& Bagnulo, S. 2008, A\&A, 480, 811

Savanov, I., \& Hubrig, S. 2003, A\&A, 410, 299

Shulyak, D., Tsymbal, V., Ryabchikova, T., Stutz, Ch., \& Weiss, W. W. 2004, A\&A, 428, 993

Sigut, T. A. A., Landstreet, J. D., \& Shorlin, S. L. S. 2000, ApJ, 530, L89

Smith, K. C. 1995, A\&A, 297, 237

Thiam, M., Wade, G. A., LeBlanc, F., \& Khalack, V. R. 2008, Contrib. Astron. Obs. Skalnaté Pleso, 38, 461

Turcotte, S., Richer, J., \& Michaud, G. 1998, ApJ, 504, 559

Vauclair, S., \& Vauclair, G. 1982, ARA\&A, 20, 37

Wade, G. A., Ryabchikova, T. A., Bagnulo, S., \& Piskunov, N. 2001, in Magnetic Fields across the Hertzsprung-Russell Diagram, ed. G. Mathys, S. K. Solanki, \& D. T. Wickramasinghe, ASP Conf. Ser., 248, 373

Wegner, G. 1976, MNRAS, 177, 99 